\title{
Management of Cannabis Use in Breastfeeding Women: The Untapped Potential of International Board Certified Lactation Consultants
}

\author{
Kara R. Skelton, ${ }^{1}$ Sara E. Benjamin-Neelon, ${ }^{1}$ and Kelly C. Young-Wolff ${ }^{2,3}$
}

\begin{abstract}
Recent increases in maternal cannabis use, in combination with rapidly changing cannabis policies in the United States, pose a unique threat to maternal and child health. To date, 33 states and the District of Columbia (D.C.) have legalized medicinal cannabis, and 11 states and D.C. have legalized recreational cannabis. Many other states have decriminalized cannabis and are considering legalization. Recent data suggest that maternal cannabis use is increasing. Maternal cannabis use in the postpartum period, including breastfeeding women, may contribute to negative health outcomes in young children. Perinatal health professionals should work collaboratively to safeguard maternal and child health outcomes from potential adverse health effects of cannabis use. To that end, we highlight the critical role International Board Certified Lactation Consultants ${ }^{\circledR}$ (IBCLCs ${ }^{\circledR}$ ) could play in postpartum management of cannabis use. IBCLCs have direct access to a large number of women shortly after delivery and throughout the postpartum period. They are an extremely well-positioned, but arguably underutilized, and are potential partners for postpartum management of cannabis use. Given the rapport IBCLCs frequently develop with their patients, these mothers may be especially willing to disclose cannabis use and be receptive to education and advice to quit cannabis use while breastfeeding. As such, we propose that the role of IBCLCs be promoted to support families with nonpunitive education about the potential risks, advice to not use cannabis while breastfeeding, and timely referral for treatment, when needed. This approach holds promise for improving the health and quality of life for breastfeeding women and their infants.
\end{abstract}

Keywords: lactation, marijuana, management

\section{Introduction}

C ANNABIS USE BY breastfeeding women is a growing concern in the United States. A number of national obstetric, pediatric, and breastfeeding advocacy organizations have put forth recommendations encouraging clinical care providers to screen women for cannabis use during the prenatal, perinatal periods, and postpartum periods. ${ }^{1-3}$ However, these recommendations largely fail to mention a key potential partner-International Board Certified Lactation Consultants ${ }^{\circledR}$ (IBCLCs $\left.{ }^{\circledR}\right)$. In addition to recommendations about abstaining from cannabis use during pregnancy, these recommendations state that women should abstain from using any cannabis products while breastfeeding, ${ }^{1,3}$ be informed about the lack of data on the safety of use while breastfeeding, ${ }^{4}$ and be educated about potential risks of cannabis use during breastfeeding for infants, ${ }^{1-3}$ while advocating for continued breastfeeding. ${ }^{2}$ IBCLCs are well positioned to implement and advance these recommendations. In this speaking out, we argue that IBCLCs are an underutilized partner in the management of maternal cannabis use and education throughout the postpartum period because they have regular and consistent contact with breastfeeding women. Further, the involvement of IBCLCs in this process may increase disclosure of cannabis use due to the trust and rapport they often establish with women. Ultimately, we call upon national organizations to include training for IBCLCs in the nonpunitive management of maternal cannabis use, evidence-based cannabis education, and referral for cannabis use treatment, as needed. Encouraging perinatal care providers to partner with IBCLCs to implement clinical cannabis recommendations may help protect infants and other children in the home from potential adverse health consequences associated with maternal cannabis use. This is especially important and timely, as more U.S. states legalize or consider legalizing cannabis.

\footnotetext{
${ }^{1}$ Department of Health, Behavior and Society, Johns Hopkins Bloomberg School of Public Health, Baltimore, Maryland.

${ }^{2}$ Division of Research, Kaiser Permanente Northern California, Oakland, California.

${ }^{3}$ Department of Psychiatry, Weill Institute for Neurosciences, University of California, San Francisco, California.
} 


\section{A Changing Landscape for Cannabis}

Currently, cannabis is federally classified as a Schedule I drug, meaning that it has no accepted medical uses and a high potential for abuse. ${ }^{5}$ Despite this, 11 U.S. states and the District of Columbia have legalized cannabis for both medicinal and adult recreational uses, and an additional 22 states have legalized cannabis for medical use only. Given the current momentum of cannabis legalization in the United States, more states are anticipated to legalize both medicinal and recreational cannabis over the next several years. Increasing legalization of cannabis, commercialization, and rising $\Delta$-9-tetrahydrocannabinol $(\Delta 9-\mathrm{THC})^{6}$ potency have potential to affect child health and development outcomes through rises in maternal perinatal cannabis use $\mathrm{e}^{7,8}$ especially for infants exposed through breast milk.

\section{Cannabis Use While Breastfeeding Is an Important Public Health Issue}

Cannabis exposure through breast milk raises concerns about potential adverse health effects on infant growth and neurodevelopment. ${ }^{2,9,10}$ Initial data indicate that the psychoactive substance in cannabis, THC, is easily transferred to the infant through breast milk and takes $\sim 6$ days for the drug to clear from the infant's system. ${ }^{11}$ With its high lipid solubility and low molecular weight, THC is rapidly stored in lipidfilled tissues of the infant, such as the brain. ${ }^{2}$ Higher THC breast milk concentrations are associated with greater frequency of maternal cannabis use, ${ }^{11}$ indicating a need for intervention to avoid adverse health consequences of cannabis exposure through breast milk. A recent systematic review found that the number of studies showing adverse effects of cannabis exposure during lactation outweighs the number of studies showing no association. ${ }^{4}$ There have also been recent changes in the availability and route of exposure (e.g., smoking, ingestion) in recent years. ${ }^{12}$ As a result, there is a need for contemporary studies on the pharmacokinetics and acute and chronic health effects of cannabis exposure through breast milk that account or use frequency and mode of cannabis administration. However, in the absence of sufficient data, national guidelines discourage cannabis use during breastfeeding, ${ }^{1-3}$ and recommend that women be educated on possible risks of cannabis exposure, including long-term neurobehavioral effects ${ }^{2}$ and secondhand smoke exposure. ${ }^{3}$ In addition, limitations of existing data regarding cannabis use during breastfeeding, in combination with the immense benefits of continued breastfeeding for both woman and infants, have led breastfeeding organizations to recommend continuation of breastfeeding, while minimizing cannabis use as much as possible. ${ }^{2}$ This type of lactation management would be relatively straightforward to incorporate into the existing scope of IBCLCs, which could mitigate potential adverse health consequences of maternal cannabis use.

Despite these potential risks and national recommendations to avoid cannabis use while breastfeeding, women are using cannabis during the postpartum period. Ko et al. used 2009-2011 data from the Pregnancy Risk Assessment Monitoring System to examine postpartum cannabis use in two states without legalized recreational cannabis. They found that postpartum cannabis use prevalence was $6.8 \%$ in Alaska and Vermont combined. ${ }^{13}$ Next, in a cross-sectional study of women from Colorado who used cannabis (where recreational cannabis is legal), $88.6 \%$ initiated breastfeeding and $64.4 \%$ of these women reporting sustained breastfeeding for $\geq 9$ weeks. ${ }^{14}$ However, another study conducted in states without legalized recreational cannabis found that cannabis users were less likely to breastfeed $>8$ weeks $(18.1 \%$ in cannabis users versus $34.9 \%$ in noncannabis users). ${ }^{13}$ Cannabis legalization and social media claims of safety of cannabis use during the postpartum period ${ }^{3}$ may contribute to women's perceptions that cannabis use while breastfeeding is safe. ${ }^{4}$ IBCLCs are extremely well positioned for the clinical management of cannabis use in the postpartum period through nonpunitive and evidence-based education.

\section{International Board Certified Lactation Consultants}

IBCLCs are specialized health professionals who complete didactic and clinical training, and provide women with evidence-based counseling for the clinical management of breastfeeding, including breastfeeding cessation. ${ }^{15}$ Currently, there are 17,389 International Board Certified Lactation Consultants in the United States. ${ }^{16}$ IBCLCs work in a variety of settings, including hospitals, pediatrician offices, in private practice, and within the Special Supplemental Nutrition Program for Women, Infants, and Children. In the settings, IBCLCs provide counseling and support to diverse women to meet their breastfeeding goals. Women have found breastfeeding support from their primary health care provider to be insufficient, ${ }^{17-19}$ leading to increased demand for quality lactation support. IBCLCs are the "go-to" health professionals for all things breastfeeding, including initiation, breastfeeding issues (e.g., engorgement, cracked nipples, medication contraindications), and weaning. ${ }^{20-22}$

IBCLCs have demonstrated success facilitating and supporting breastfeeding. Women who use IBCLCs have significantly higher rates of exclusively breastfeeding at 6 months and breastfeeding at 12 months, ${ }^{23}$ which has helped national and state organizations and leaders recognize the value of IBCLCs. The U.S. Surgeon General's Call to Action to Support Breastfeeding recommends 8.6 International Board Certified Lactation Consultants per 1,000 live births. ${ }^{24}$ In addition, under the Affordable Care Act of 2010, ${ }^{25}$ all new health plans must cover up to six visits addressing "comprehensive prenatal and postnatal lactation support [and] counseling" without a copayment or other cost sharing. The large demand for IBCLCs by postpartum women and expanded coverage of IBCLCs for breastfeeding support under new insurance plans make them an ideal conduit for management of maternal cannabis use.

\section{Untapped Potential for IBCLCs}

Most of the national recommendations encourage obstetricians, gynecologists, and pediatricians to screen postpartum women for cannabis use. ${ }^{1-3}$ However, these clinical care providers have few opportunities to do so. In fact, as many as $40 \%$ of women do not attend a single postpartum visit. ${ }^{21}$ For women who do attend, most postpartum care appointments are $\sim 25$ minutes in duration. ${ }^{26}$ Topics covered by the postpartum visit include mood and emotional well-being, infant care and feeding, sexuality and contraception, sleep and fatigue, physical recover from birth, chronic disease management, and health maintenance. ${ }^{21}$ This long list of covered domains may not leave adequate time to address cannabis screening and education. In addition, the extent to which clinical care 
providers implement these recommendations is not yet known. In the meantime, IBCLCs can partner with obstetricians and pediatricians, presenting a united front to address cannabis use during breastfeeding.

In addition, IBCLCs are extremely well positioned during the perinatal and postpartum periods to address cannabis use among breastfeeding women because they often earn the trust and confidence of their patients. They are able to establish relationships with women early after birth and maintain this relationship through multiple points of contact with women after the conclusion of obstetric care 12 weeks after birth. ${ }^{21}$ Due to the sensitive nature of breastfeeding, in combination with the ongoing personal and emotional support provided to women, there is often a deep, intimate relationship established between women and IBCLCs. ${ }^{27}$ Further, IBCLC consultations average $~ 45-60$ minutes in length ${ }^{28}$ - about twice the length of the average postpartum obstetric appointment. ${ }^{26}$ As established rapport and feelings of safety have led victims of child sexual abuse to disclose past abuse to IBCLCs, ${ }^{28}$ it is plausible to think that this established rapport may increase mother's disclosure of cannabis use. This intimate relationship, including established trust, may lead women to feel more comfortable asking questions about cannabis use while breastfeeding, for both current cannabis users and women considering cannabis use. Combined, these points show that IBCLCs are extremely well positioned to clinically manage maternal cannabis use, provide women with data-based information about the potential risks of cannabis use during breastfeeding, and advise breastfeeding women to abstain from or minimize cannabis use, per clinical recommendations. ${ }^{1-3}$

\section{Call to Action}

With a large push at the national level for increased promotion of breastfeeding, in combination with state legalization of cannabis, we recommend the role of IBCLCs in the clinical management of maternal cannabis use be highlighted and expanded. IBCLCs are in a unique position to communicate the potential risks of cannabis use while breastfeeding in nonpunitive language. They can also help interpret current evidence in a meaningful way. Simultaneously, IBCLCs can advocate for continued breastfeeding and cessation of cannabis use, per national guidelines. ${ }^{1,2}$

The clinical management of cannabis use by IBCLCs should encompass maternal cannabis use frequency and modes of administration (e.g., smoking, ingestion), providing specific information about potential acute and chronic adverse health effects for both the woman and infant. This should also include questions regarding the couse of other substances, such as alcohol and tobacco, as they have been linked with cannabis use. $^{29}$ In addition, IBCLCs should inquire about cannabis use by other family members and storage of cannabis use in the home. These types of questions could organically lead to discussions about how to safely store cannabis in the home, which could protect children from THC toxicity through accidental ingestion of cannabis-containing products. When discussing cannabis use, it is imperative that these discussions are not be used to stigmatize or penalize breastfeeding women. Instead, this should be viewed as an opportunity to provide education about the potential risks, brief advice to quit, and referrals to substance use treatment, as needed. Thus, IBCLCs should be well trained on how to provide sensitive and nonjudgmental management of maternal cannabis use and meaningful education, so that women do not become fearful of lactation visits, but instead continue to see them as a safe opportunity to discuss cannabis and disclose use.

IBCLCs can play an important role in conveying accurate information to breastfeeding women about the potential harm cannabis use may have on their infants, while advocating for continuation of breastfeeding. However, there is evidence that IBCLCs need additional training on cannabis use and safety. ${ }^{30}$ A recent survey of lactation professionals found that $\sim 41 \%$ reported recommending continuing cannabis use while breastfeeding, which goes against current clinical recommendations. ${ }^{30}$ This underlines the need to educate IBCLCs about current clinical recommendations and potential harms of cannabis exposure through breast milk and train them to encourage breastfeeding, while advising mothers to abstain from and minimize cannabis use. The clinical management of maternal cannabis use, including education, should be a topic area covered as part of the certification process for IBCLCs.

Therefore, we call upon breastfeeding organizations, such as the Academy for Breastfeeding Medicine and the United States Lactation Consultant Association, along with certifying organizations, such as the International Board of Lactation Consultant Examiners, to create and disseminate cannabisrelated education and training materials. These materials should be focused on addressing postpartum maternal cannabis use, use of cannabis in the home, and safe storage of cannabis. Training in the clinical management of cannabis use should be incorporated as part of standard certification programs. Existing patient handouts, such as those developed by the Colorado Department of Public Health and Environment, could be modified for use by IBCLCs. ${ }^{31}$ Lactation support visits could also provide an important opportunity to educate women about unintentional consequences of cannabis use and storage in the home, including child THC toxicity through accidental ingestion, secondhand smoke exposure, and the risks associated with driving or taking care of children while using cannabis. Therefore, IBCLCs should also be trained on how to educate women on additional mechanisms of postpartum cannabis exposure and associated potential consequences. To ensure that children are safeguarded from potential adverse health effects of cannabis exposure, training for IBCLCs on the clinical management of maternal cannabis use, including referral to treatment, as needed, and education should begin now and be based on existing scientific evidence.

\section{Conclusion}

Increasing momentum for state cannabis legalization, commercialization, and rising THC potency have the potential to affect child health and development through maternal postpartum use-especially for infants who are exposed through breast milk. As such, there is an urgent need for clinical care providers and IBCLCs to unite to safeguard children's health. IBCLCs establish strong relationships with breastfeeding women during the postpartum period, making them an ideal, but underutilized partner in the clinical management of cannabis, which includes referral to treatment for cannabis use disorder, as needed, and data-driven education. Thus, it is important that the scope of practice for IBCLCs be revisited to include clinical management of cannabis use. This type of support must be conducted in a nonpunitive manner, and we recommend standardized training for IBCLCs through 
national breastfeeding organizations, as well as certifying bodies. IBCLCs are well poised to aid in the clinical management of cannabis use and education in a sensitive and nonjudgmental manner, which includes referral to addiction treatment, as needed. Engaging and empowering IBCLCs in this manner have high potential to improve the health and quality of life for both breastfeeding women and their infants.

\section{Disclosure Statement}

No competing financial interests exist.

\section{Funding Information}

This study was supported, in part, by an NIH NIDA K01 Award (DA043604). The funding source was not involved in the conceptualization or drafting of this viewpoint.

\section{References}

1. American College of Obstetricians and Gynecologists, Committee on Obstetric Practice. Committee Opinion No. 722: Marijuana use during pregnancy and lactation. Obstet Gynecol 2017;130:e205-e209.

2. Reece-Stremtan S, Marinelli KA; and Academy of Breastfeeding Medicine. ABM clinical protocol \#21: Guidelines for breastfeeding and substance use or substance use disorder, revised 2015. Breastfeed Med 2015;10:135-141.

3. Ryan SA, Ammerman SD, O'Connor ME. Marijuana use during pregnancy and breastfeeding: Implications for neonatal and childhood outcomes. Pediatrics 2018;142:e20181889.

4. Mourh J, Rowe H. Marijuana and breastfeeding: Applicability of the current literature to clinical practice. Breastfeed Med 2017;12:582-596.

5. Blatman M. The Controlled Substances Act of 1970. Penn Med 1971;74:45-46.

6. Mehmedic Z, Chandra S, Slade D, et al. Potency trends of $\triangle 9$-THC and other cannabinoids in confiscated cannabis preparations from 1993 to 2008. J Forensic Sci 2010;55: 1209-1217.

7. Wang GS. Pediatric concerns due to expanded cannabis use: Unintended consequences of legalization. J Med Toxicol 2017;13:99-105.

8. Jansson LM, Bunik M, Bogen DL. Lactation and the marijuana-using mother. Breastfeed Med 2015;10:342-343.

9. Volkow ND, Compton WM, Wargo EM. The risks of marijuana use during pregnancy. JAMA 2017;317:129-130.

10. Wilson KM, Torok MR, Wei B, et al. Detecting biomarkers of secondhand marijuana smoke in young children. Pediatr Res 2017;81:589.

11. Bertrand KA, Hanan NJ, Honerkamp-Smith G, et al. Marijuana use by breastfeeding mothers and cannabinoid concentrations in breast milk. Pediatrics 2018;142:e20181076.

12. Amirshahi MM, Moss MJ, Smith SW, et al. ACMT Position Statement: Addressing pediatric cannabis exposure. J Med Toxicol 2019;15:212-214.

13. Ko JY, Tong VT, Bombard JM, et al. Marijuana use during and after pregnancy and association of prenatal use on birth outcomes: A population-based study. Drug Alcohol Depend 2018;187:72-78.

14. Crume TL, Juhl AL, Brooks-Russell A, et al. Cannabis use during the perinatal period in a state with legalized recreational and medical marijuana: The association between maternal characteristics, breastfeeding patterns, and neonatal outcomes. J Pediatr 2018;197:90-96.
15. Association ILC. What is an IBCLC. Www.ilca.org/whyibclc/ibclc (accessed September 18, 2019).

16. IBCLE. Current Statistics on Worldwide IBCLCs 2019. Updated 2019. https://iblce.org/about-iblce/current-statisticson-worldwide-ibclcs (accessed September 18, 2019).

17. Johnson AM, Kirk R, Rooks AJ, et al. Enhancing breastfeeding through healthcare support: Results from a focus group study of African American mothers. Matern Child Health J 2016;20:92-102.

18. Meek JY. Pediatrician competency in breastfeeding support has room for improvement. Pediatrics 2017;140:e20172509.

19. Feldman-Winter L, Szucs K, Milano A, et al. National trends in pediatricians' practices and attitudes about breastfeeding: 1995 to 2014. Pediatrics 2017;140:e20171229.

20. Chetwynd EM, Wasser HM, Poole C. Breastfeeding support interventions by International Board Certified Lactation Consultants: A systemic review and meta-analysis. J Hum Lact 2019;35:424-440.

21. American College of Obstetricians and Gynecologists, Committee on Obstetric Practice. Committee Opinion No. 666: Optimizing postpartum care. Obstet Gynecol 2016;127:e187.

22. Torres JM. Medicalizing to demedicalize: Lactation consultants and the (de) medicalization of breastfeeding. Soc Sci Med 2014;100:159-166.

23. Patel S, Patel S. The effectiveness of lactation consultants and lactation counselors on breastfeeding outcomes. J Hum Lact 2016;32:530-541.

24. US Department of Health and Human Services. The Surgeon General's call to action to support breastfeeding 2011. https://www.ncbi.nlm.nih.gov/pubmed/21452448

25. Kapinos KA, Bullinger L, Gurley-Calvez T. Lactation support services and breastfeeding initiation: Evidence from the Affordable Care Act. Health Serv Res 2017;52:2175-2196.

26. American College of Obstetricians and Gynecologists. Coding for Postpartum Services. 2018. https://www.acog .org/-/media/Departments/Toolkits-for-Health-Care-Providers/ Postpartum-Toolkit/ppt-coding.pdf?dmc $=1 \&$ ts $=20191104 \mathrm{~T}$ 1913428012 (accessed October 11, 2019).

27. Burns E, Schmied V. "The right help at the right time": Positive constructions of peer and professional support for breastfeeding. Women Birth 2017;30:389-397.

28. Wambach K, Spencer B. Breastfeeding and Human Lactation. Burlington, MA: Jones \& Bartlett Publishers, 2019.

29. Agrawal A, Rogers CE, Lessov-Schlaggar CN, et al. Alcohol, cigarette, and cannabis use between 2002 and 2016 in pregnant women from a nationally representative sample. JAMA Pediatr 2019;173:95-96.

30. Bergeria CL, Heil SH. Surveying lactation professionals regarding marijuana use and breastfeeding. Breastfeed Med 2015;10:377-380.

31. Colorado Department of Public Health and Environment. Marijuana Pregnancy \& Breastfeeding Guidance 2017. www.colorado.gov/pacific/sites/default/files/MJ_RMEP_ Pregnancy-Breastfeeding-Clinical-Guidelines.pdf (accessed September 18, 2019).

Address correspondence to: Kara R. Skelton, PhD

Department of Health, Behavior and Society Johns Hopkins Bloomberg School of Public Health 624 N. Broadway Baltimore, MD 21205

E-mail: kara.skelton@jhu.edu 